\title{
Circulating miR-31-5p may be a potential diagnostic biomarker in nasopharyngeal carcinoma
}

\author{
S. J. YI ${ }^{1, *}$, P. LIU ${ }^{2, *}$, B. L. CHEN ${ }^{3}$, L. OU-YANG ${ }^{3}$, W. M. XIONG ${ }^{4}$, J. P. SU ${ }^{1, *}$
}

${ }^{1}$ Department of Otolaryngology Head and Neck Surgery, First Affiliated Hospital of Guangxi Medical University, Guangxi Medical University, Nanning, China; 'Department of Otolaryngology Head and Neck Surgery, Affiliated Hospital of Guilin Medical University, Guilin, China; ${ }^{3}$ Department of Otolaryngology Head and Neck Surgery, The Second Xiangya Hospital of Central South University, Central South University, Changsha, Hunan, China; ${ }^{4}$ Department of Otolaryngology Head and Neck Surgery, People's Hospital of Guangxi Zhuang Autonomous Region, Nanning, China

${ }^{*}$ Correspondence: glmcysj@163.com

"Contributed equally to this work.

Received November 9, 2018 / Accepted March 13, 2019

\begin{abstract}
Aberrant expression of miR-31-5p has been detected in various cancers and plays a significant role in tumorigenesis. Low miR-31-5p expression was present in nasopharyngeal carcinoma (NPC) tissues and cell lines and acted as a tumor suppressive miRNA. Currently, circulating miRNAs are emerging as novel biomarkers for the early diagnosis of cancers using a non-invasive method. However, circulating miR-31-5p has rarely been reported in NPC. Therefore, the aim of this study was to explore peripheral blood miR-31-5p levels as a noninvasive biomarker and evaluate its clinical value for the early diagnosis of patients with NPC. A total of 110 participants were recruited, including 55 NPC patients and 55 healthy controls. Peripheral blood samples were collected from these participants, and total RNA was extracted to quantify the relative expression of miR-31-5p by RT-qPCR. We found a significantly lower expression of miR-31-5p in the NPC patients than in the healthy controls. Furthermore, low expression of miR-31-5p was highly correlated with tumor-node-metastasis (TNM) stage (I+II vs III+IV, p=0.001), T classification (T1 vs T2+T3+T4, p=0.036) and local lymph node metastasis (N1$\mathrm{N} 3$ vs N0, $\mathrm{p}=0.002)$, but not distant metastasis $(\mathrm{p}=0.288)$. Moreover, miR-31-5p showed a moderate diagnostic performance $(\mathrm{AUC}=0.866$, sensitivity $=0.782$, specificity $=0.818)$. Thus, we concluded that circulating miR-31-5p can be a potentially novel and non-invasive biomarker for the early diagnosis of NPC and an attractive therapeutic target in NPC patients.
\end{abstract}

Key words: nasopharyngeal carcinoma, circulating miR-31-5p, diagnostic, biomarker

Nasopharyngeal carcinoma is a malignant head and neck tumor that is prevalent in southern China, particularly in the Guangxi Province, Guangdong Province and Hunan Province [1-3]. Although epidemiological trends suggest that the incidence of nasopharyngeal carcinoma is gradually declining and the mortality rates of the disease have fallen substantially (even in endemic regions) in the past decade [4], the survival rate is still dissatisfactory due to locoregional recurrence and distant metastasis, especially in advancedstage cases. Therefore, early diagnosis is particularly important. Nasal endoscopic biopsy, as an invasive method is commonly used to diagnose NPC, but in early-stage cases, it is difficult to obtain lesion tissue. Therefore, non-invasive and cancer-specific biomarkers for early diagnosis are needed for NPC patients.

MiRNAs represent a family of small non-protein-coding RNAs that consist of 20-24 nucleotides and that regulate the expression of numerous target genes at the post-transcriptional level [5]. Accumulating evidence has demonstrated that miRNAs play an important role in cancer by acting as tumor suppressors or oncogenes [6-7]. In addition, in many studies miRNAs have been regarded as promising prognostic biomarkers and novel therapeutic targets and have also been developed as biomarkers for earlier diagnosis of cancer as an alternative to the existing methods that are mostly based on tissue detection [8]. However, not all patients could provide a specimen for early diagnosis, particularly in the early stage when cancerous tissue can hardly be distinguished from normal tissue. Recently, a diagnostic method based on the expression profile of miRNAs in body fluid samples, such as whole blood, saliva and digestive juice, has attracted considerable attention [9]. In addition, studies have demonstrated that cancer cells contribute a pool of miRNAs that could be detected in the patients' circulation due to their stability 
[10,11]. Therefore, the application of circulating miRNA alone or in combination with other biomarkers might be beneficial for the early detection of NPC.

MiR-31-5p, as one of the most popular candidates, has been reported in different types of cancers. Zhao et al. showed that miR-31-5p was downregulated in hepatocellular carcinoma (HCC) cell lines compared with normal liver cell lines. The overexpression of miR-31-5p inhibited HCC cell growth, migration, invasion and cell cycle arrest induced by targeting specific protein 1 (SP1) [12]. Igarashi et al. reported that miRNA-31-5p might regulate BRAF activation and play a role in the signaling pathway downstream of EGFR in colorectal cancer. Further research found that miR-31-5p might be a useful and additional prognostic biomarker for anti-EGFR therapy [13]. Cheung et al. demonstrated that miR-31-5p functions as an important tumor suppressive miRNA in NPC and demonstrated that miR-31-5p decreased NPC cell growth by directly regulating hypoxia-inducible factor 1 (FIH1) and minichromosome maintenance protein 2 (MCM2) [14]. Moreover, Wu et al. first used a non-viral minicircle vector targeting the expression of miR-31-5p and demonstrated that minicircle-oriP-miR-31 can serve as a novel targeted miRNA therapy approach for nasopharyngeal carcinoma [15]. These data further clarified that miR-31-5p may be a promising candidate for comprehensive investigations and a possible site for gene therapy in the future.

Since miR-31-5p acts as a tumor suppressor gene in NPC and repressed miR-31-5p expression can promote tumorigenesis [14], we hypothesized that miR-31-5p could be detectable as a biomarker in patients. Then, the study was conducted to explore the expression of circulating miR-31-5p in peripheral blood samples and to evaluate its clinical diagnostic value in NPC patients.

\section{Patients and methods}

Patients and samples. A total of 55 patients who were pathologically diagnosed with NPC were enrolled at the Affiliated Hospital of Guilin Medical University between February 2015 and December 2016. The NPC patients included 35 males and 20 females with a median age of 50 years (range 29-70 years). The control group consisted of 31 males and 24 females with a median age of 45 years (range 35-69 years) who were recruited from the physical examination center and who did not have tumors or other diseases. Peripheral blood samples were collected before any intervention. Tumor staging was classified according to the AJCC (the seventh version). All of the participants involved in this study were informed about the study and signed a consent form. Our study was approved by the ethics committee of the Affiliated Hospital of Guilin Medical University and complied with the Declaration of Helsinki.

RNA extraction and quantitative reverse transcription (qRT-PCR). Total RNA was isolated from peripheral blood samples with TRI Reagent BD (Molecular Research Center, Cincinnati, USA). The concentration of total RNA was quantitated by measuring the absorbance at $260 \mathrm{~nm}$, followed by reverse transcription into cDNA with oligo-dT primers (Invitrogen, USA) according to the manufacturer's instructions. Reverse transcription was performed based on the instructions from the Gene Amp system 9700 (Applied Biosystems). The RT mix included total RNA $200 \mathrm{ng}, 1 \mu \mathrm{l}$ $(1 \mu \mathrm{M})$ of RT special primer, $2 \mu \mathrm{l}(2.5 \mathrm{mM})$ of dNTP Mix, $0.3 \mu \mathrm{l}(40 \mathrm{U} / \mu \mathrm{l})$ of RNase inhibitor, $2 \mu \mathrm{l}$ of 10x RT Buffer $(250$ $\mathrm{mM}$ Tris- $\mathrm{HCl}, \mathrm{pH} 8.3,200 \mathrm{mM} \mathrm{KCl}, 40 \mathrm{mM} \mathrm{MgCl}_{2}, 5 \mathrm{mM}$ DTT), $2 \mu \mathrm{l}(10 \mathrm{U} / \mu \mathrm{l})$ of Transcript-MLV, and RNase-free $\mathrm{H}_{2} \mathrm{O}$ to a total volume of $20 \mu \mathrm{l}$. The reaction conditions were
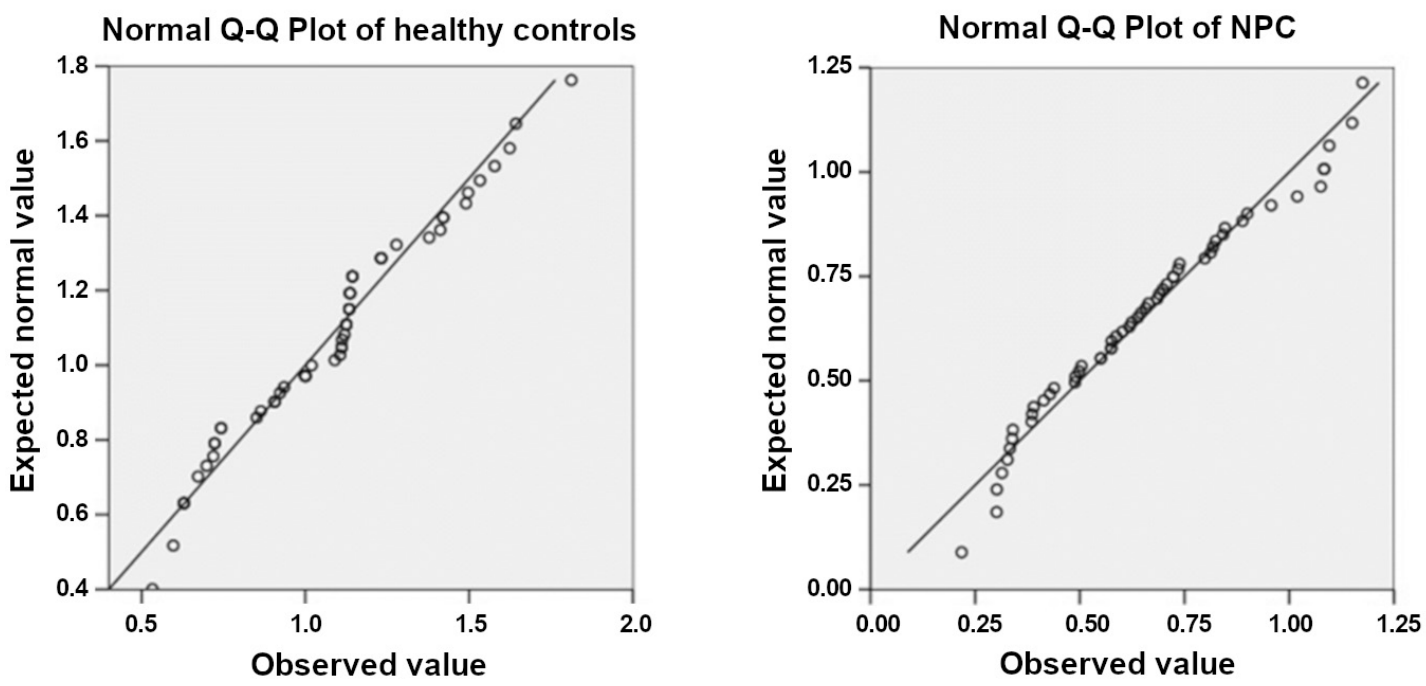

Figure 1. Testing distribution of data in NPC and healthy control groups by Q-Q plot. The results showed that data were normally distributed in both NPC and healthy control groups. 
as follows: $16^{\circ} \mathrm{C}$ for 30 minutes, $42^{\circ} \mathrm{C}$ for 42 minutes, and $85^{\circ} \mathrm{C}$ for 5 minutes. qRT-PCR using cDNA as a template was performed using the Vii7 Real-time PCR System (Applied Biosystems). U6 was used as an internal control. $10 \mu \mathrm{l}$ reaction mix consisted of $5 \mu \mathrm{l}$ of $2 \times$ Master Mix, $0.5 \mu \mathrm{l}(10 \mu \mathrm{M})$ of forward primer, $0.5 \mu \mathrm{l}(10 \mu \mathrm{M})$ of reverse primer, $2 \mu \mathrm{l}$ of cDNA and $2 \mu \mathrm{l}$ of distilled water. The threshold cycle (CT) was set within the exponential phase of the PCR. The relative expression levels were calculated and quantified by using the $2^{-\Delta \Delta C T}$ method after normalization. The primer sequences were as follows: U6: F: 5'GCTTCGGCAGCACATATACTAAAAT3', R: 5'CGCTTCACGAATTTGCGTGTCAT3'; and miR-31-5p: GSP: 5'GGAGGCAAGATGCTGGC3', R: 5'GTGCGTGTCGTGGAGTCG3'. The experiment was performed in triplicate.

Statistical analysis. Data were normally distributed (Figure 1) and are presented in the form of the mean \pm SD. We used Student's t-test and one-way ANOVA to determine the differences in the expression level of miRNA between groups classified by the clinical characteristics of the patients. An ROC curve was generated and the area under the curve was calculated to assess the feasibility of utilizing miRNA expression as a diagnostic biomarker to distinguish NPC patients from healthy people. A multivariate linear regression approach was used to analyze the correlation of miR-31-5p with age, gender and disease status (with or without NPC). A $\mathrm{p}$-value of $<0.05$ was considered statistically significant. The statistical analysis was performed with SPSS 18.0.

\section{Results}

The expression of miR-31-5p in peripheral blood samples of NPC patients and healthy controls. The results showed that there was a significant difference between the NPC patients and the healthy subjects. The NPC patient group expressed a higher level of miR-31-5p than the healthy control group (Table 1, Figure 2). Multivariate linear regression analysis for age, gender and disease status on miR-31-5p expression level is shown in Table 2. It could be observed from the model summary that there were two models, modell and model2. According to the model fitting effect, model $1\left(\mathrm{R}^{2}=0.380\right)$ was a little better than the model 2
$\left(\mathrm{R}^{2}=0.371\right)$. Therefore, gender $(\mathrm{p}=0.875)$ and age $(\mathrm{p}=0.520)$ variables were excluded and generated an equation as: $Y=1.882-0.430{ }^{\star} X 1 \quad(Y$, miR-31-5p expression level; $X 1$, disease status). These results indicated that miR-31-5p expression level was only related to the disease status, regardless of gender or age.

Decreased expression of circulating miR-31-5p is correlated with the clinicopathological characteristics of NPC. To study the importance of miR-31-5p expression, clinical relationship between miR-31-5p expression and clinicopathological parameters in patients with NPC was further

Table 1. The baseline clinical characteristics of the NPC patients and healthy controls.

\begin{tabular}{lccc}
\hline Variables & NPC & Healthy & p-value \\
\hline Male/Female & $21 / 34$ & $25 / 30$ & \\
Age (Y) & $49.3 \pm 11.8$ & $48.3 \pm 10.5$ & \\
miR-31-5p expression & $0.651 \pm 0.246$ & $1.083 \pm 0.298$ & $<0.0001^{\mathrm{a}}$ \\
\hline
\end{tabular}

Note: The NPC patient group expressed a higher level of miR-31-5p than the healthy control group $(\mathrm{p}<0.0001)$. a, Student's test.

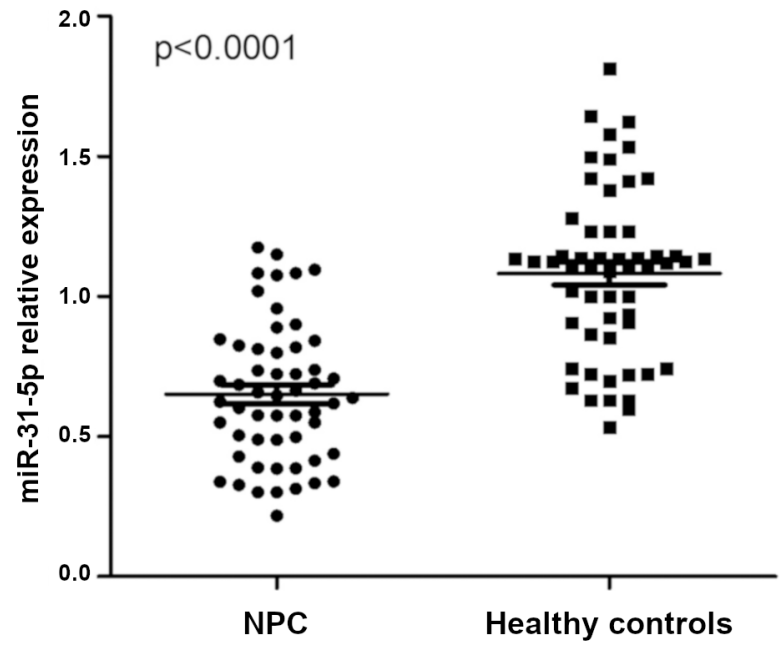

Figure 2. qRT-PCR quantified the miR-31-5p expression in NPC patients and healthy controls. There was a significant difference between the NPC patients and the healthy controls $(\mathbf{p}<0.0001)$.

Table 2. Multivariate linear regression analysis for age, gender and disease status on miR-31-5p expression level.

\begin{tabular}{|c|c|c|c|c|c|c|c|}
\hline \multirow[t]{2}{*}{ Model } & \multirow{2}{*}{$\begin{array}{l}\text { Adjusted } \\
\text { R Square }\end{array}$} & & \multicolumn{2}{|c|}{ Unstandardized coefficients } & \multirow{2}{*}{$\begin{array}{c}\text { Standardized } \\
\text { coefficients }\end{array}$} & \multirow[t]{2}{*}{$\mathbf{t}$} & \multirow[t]{2}{*}{ p-value } \\
\hline & & & B & Std. Error & & & \\
\hline \multirow[t]{2}{*}{1} & 0.380 & (constant) & 1.882 & 0.037 & & 29.287 & $<0.001$ \\
\hline & & $\mathrm{X} 1$ & -0.430 & 0.052 & -0.621 & -8.240 & $<0.001$ \\
\hline \multirow[t]{4}{*}{2} & 0.371 & (constant) & 1.073 & 0.050 & & 21.371 & $<0.001$ \\
\hline & & $\mathrm{X} 1$ & -0.430 & 0.053 & -0.621 & -8.157 & $<0.001$ \\
\hline & & $\mathrm{X} 2$ & -0.009 & 0.054 & -0.012 & -0.158 & 0.875 \\
\hline & & $\mathrm{X} 3$ & -0.036 & 0.055 & 0.050 & 0.645 & 0.520 \\
\hline
\end{tabular}

Note: dependent variable, Y (miR-31-5p expression level); X1 (disease status (with or without NPC)); X2 (age); X3 (gender). 
Table 3. The expression of miR-31-5p in peripheral blood samples and clinical characteristics of NPC patients.

\begin{tabular}{|c|c|c|c|}
\hline $\begin{array}{l}\text { Clinical } \\
\text { characteristics }\end{array}$ & Samples (n) & $\begin{array}{l}\text { Relative expression } \\
\text { of miR-31-5p }\end{array}$ & p-value \\
\hline \multicolumn{4}{|l|}{ Gender } \\
\hline Male & 21 & $0.610 \pm 0.222$ & \multirow{2}{*}{$0.121^{\mathrm{a}}$} \\
\hline Female & 34 & $0.717 \pm 0.265$ & \\
\hline \multicolumn{4}{|l|}{ Age } \\
\hline$<50$ & 27 & $0.745 \pm 0.318$ & \multirow{2}{*}{$0.149^{\mathrm{a}}$} \\
\hline$\geq 50$ & 28 & $0.632 \pm 0.268$ & \\
\hline \multicolumn{4}{|l|}{ T classification } \\
\hline $\mathrm{T} 1$ & 6 & $0.906 \pm 0.125$ & \multirow{4}{*}{$0.036^{\mathrm{b}}$} \\
\hline $\mathrm{T} 2$ & 9 & $0.667 \pm 0.322$ & \\
\hline $\mathrm{T} 3$ & 21 & $0.643 \pm 0.236$ & \\
\hline $\mathrm{T} 4$ & 19 & $0.576 \pm 0.205$ & \\
\hline \multicolumn{4}{|c|}{ Lymph node status } \\
\hline No & 7 & $0.923 \pm 0.172$ & \multirow{4}{*}{$0.002^{\mathrm{b}}$} \\
\hline N1 & 2 & $0.734 \pm 0.109$ & \\
\hline $\mathrm{N} 2$ & 35 & $0.642 \pm 0.223$ & \\
\hline N3 & 11 & $0.409 \pm 0.241$ & \\
\hline \multicolumn{4}{|c|}{ Metastatic status } \\
\hline M0 & 36 & $0.677 \pm 0.238$ & \multirow{2}{*}{$0.288^{\mathrm{a}}$} \\
\hline M1 & 19 & $0.602 \pm 0.261$ & \\
\hline \multicolumn{4}{|l|}{ TNM stage } \\
\hline $\mathrm{I}+\mathrm{II}$ & 8 & $1.035 \pm 0.179$ & \multirow{3}{*}{$0.001^{\mathrm{b}}$} \\
\hline III & 17 & $0.722 \pm 0.236$ & \\
\hline IV & 30 & $0.571 \pm 0.273$ & \\
\hline
\end{tabular}

Note: a, Student's test; b one-way ANOVA test.

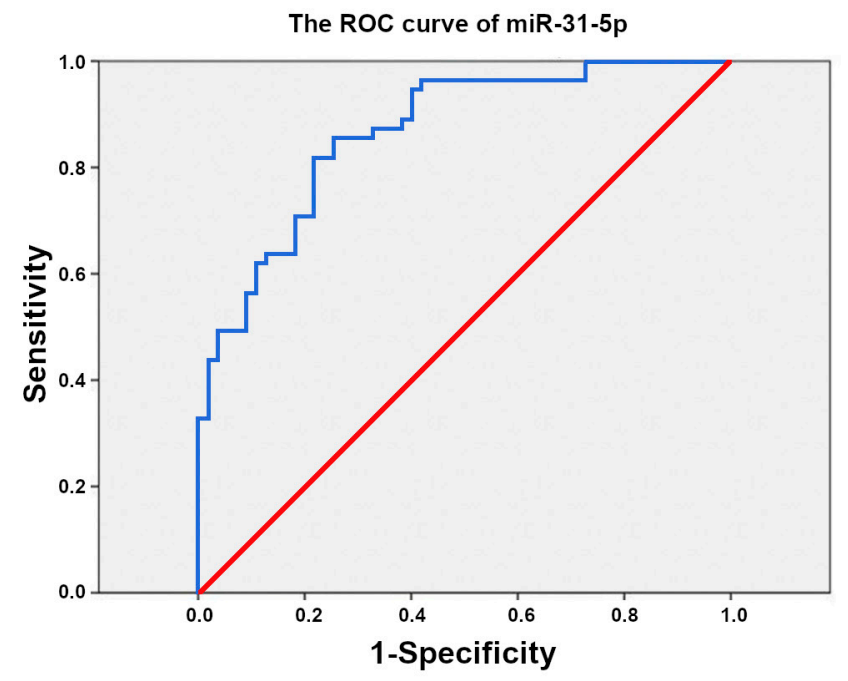

Figure 3. ROC analysis of circulating miR-31-5p in NPC patients and healthy controls: The AUC was 0.866 (95\% CI: 0.801-0.932), and the sensitivity and specificity were $78.2 \%$ (95\% CI: 65.0-88.2) and $81.8 \%(95 \%$ CI: 69.1-90.9), respectively. analyzed. The statistical analysis suggested that a low level of miR-31-5p was strongly correlated with some clinicopathological parameters in NPC patients (Table 3), including TNM stage (I+II vs III $+\mathrm{IV}, \mathrm{p}=0.001)$, T classification (T1 vs $\mathrm{T} 2+\mathrm{T} 3+\mathrm{T} 4, \mathrm{p}=0.036)$ and local lymph node metastasis (N1-N3 vs N0, $\mathrm{p}=0.002)$. However, no significant difference was observed in distant metastasis $(\mathrm{p}=0.288)$, which was likely due to the limited sample size. Additionally, there were no differences observed based on patient age and gender.

Evaluation of the diagnostic value of miR-31-5p in NPC. A receiver operating characteristic (ROC) curve was generated to evaluate the diagnostic accuracy of peripheral blood miR-31-5p, and the area under the curve (AUC) was calculated to assess the sensitivity and specificity of the peripheral blood miRNA as a diagnostic biomarker in NPC. The AUC was 0.866 (95\% CI: 0.801-0.932), and the sensitivity and specificity were $78.2 \%$ (95\% CI: $65.0-88.2$ ) and $81.8 \%$ (95\% CI: 69.1-90.9), respectively, which showed a moderate diagnostic performance (Figure 3 ).

\section{Discussion}

In this study, we first detected the expression of circulating miR-31-5p in peripheral blood samples of patients with NPC. The results showed that the expression of miR-31-5p was significantly lower in patients with NPC than in healthy controls, which exhibited a close relationship with clinical characteristics, except age, gender and distant metastasis, considering the limited sample size. In addition, detecting the expression of circulating miR-31-5p in peripheral blood samples showed a moderate diagnostic value. As mentioned above, we suggest that circulating miR-31-5p may be a potential biomarker for the early diagnosis of patients with NPC.

Currently, 2,588 different mature human miRNAs have been identified that reportedly target more than a third of the protein-coding genes [16]. MiRNA molecules play a pivotal role in virtually all cellular functions, including apoptosis, proliferation, migration and differentiation and have been identified as promising biomarkers in cancer [11, $14,16,17]$. Previously, studies were mostly tissue-based; nevertheless, a few studies were based on peripheral blood samples. Recently, several studies have reported circulating miRNA as a biomarker in cancer studies by using serum, plasma and whole blood [18-19]. For instance, Qu et al. demonstrated that circulating miR-21-5p had a relatively high diagnostic accuracy for the diagnosis of PC (sensitivity 0.77 , specificity 0.80 and an AUC 0.78 (95\% CI: $0.66-0.90)$ ) and might be a promising diagnostic biomarker [20]. A gastric cancer study revealed that miR-19b-3p and miR-16-5p might be prospective biomarkers to detect gastric cancer and have great potential in applications for early screening and progression evaluation [21]. Accordingly, we hypothesized that circulating miRNA-31-5p may also be aberrantly expressed in peripheral blood samples of 
patients with NPC. Fortunately, our results indicated that circulating miR-31-5p might act as a potential minimally invasive diagnostic biomarker for NPC.

However, there are still some limitations to our study. First, the sample size of the study was small, and larger-scale samples are needed for more profound investigations. Second, all patients were diagnosed with undifferentiated squamous cell carcinoma, and we failed to analyze the expression of miR-31-5p in different pathological types. Third, although decreased miR-31-5p levels were measured in NPC, the detailed mechanisms of miR-31-5p in tumors remain elusive. However, our relatively small case series unveiled that low expression of circulating miR-31-5p is associated with the progression of NPC and circulating miR-31-5p may provide insights into a potential minimally invasive biomarker for early diagnosis and an attractive therapeutic target for NPC patients.

Acknowledgements: This study was funded by the Guangxi Guilin Scientific Research and Technology Development Plan Project (No. 20140505-3)

\section{References}

[1] ZHU HM, JIANG XS, LI HZ, QIAN LX, DU MY et al. miR-184 Inhibits Tumor Invasion, Migration and Metastasis in Nasopharyngeal Carcinoma by Targeting Notch2. Cell Physiol Biochem 2018; 49: 1564-1576. https://doi. org/10.1159/000493459

[2] SHI X, CHEN X, LI WC, MO LJ, LIN ZY et al. Low expression of Cyfip1 may be a potential biomarker in nasopharyngeal carcinoma. Neoplasma 2018; 65: 292-295. https://doi. org/10.4149/neo_2018_170318N194

[3] FERLAY J, SOERJOMATARAM I, DIKSHIT R, ESER S, MATHERS $\mathrm{C}$ et al. Cancer incidence and mortality worldwide: sources, methods and major patterns in GLOBOCAN 2012. Int J Cancer 2015; 136: E359-386. https://doi. org/10.1002/ijc. 29210

[4] CHUA MLK, WEE JTS, HUI EP, CHAN ATC. Nasopharyngeal carcinoma. Lancet 2016; 387: 1012-1024. https://doi. org/10.1016/S0140-6736(15)00055-0

[5] TREIBER T, TREIBER N, MEISTER G. Regulation of microRNA biogenesis and its crosstalk with other cellular pathways. Nat Rev Mol Cell Biol 2019; 20: 5-20. https://doi. org/10.1038/s41580-018-0059-1

[6] LIN S, GREGORY RI. MicroRNA biogenesis pathways in cancer. Nat Rev Cancer 2015; 15: 321-333. https://doi. org/10.1038/nrc3932

[7] CALIN GA, CROCE CM. MicroRNA signatures in human cancers. Nat Rev Cancer 2006; 6: 857-866. https://doi. org/10.1038/nrc1997

[8] TURCHINOVICH A, WEIZ L, LANGHEINZ A, BURWINKEL B. Characterization of extracellular circulating microRNA. Nucleic Acids Res 2011; 39: 7223-7233. https://doi. org/10.1093/nar/gkr254
[9] PRITCHARD CC, CHENG HH, TEWARI M. MicroRNA profiling: approaches and considerations. Nat Rev Genet 2012; 13: 358-369. https://doi.org/10.1038/nrg3198

[10] LE LARGE TY, MEIJER LL, PRADO MM, KAZEMIER G, FRAMPTON AE et al. Circulating microRNAs as diagnostic biomarkers for pancreatic cancer. Expert Rev Mol Diagn 2015; 15: 1525-1529. https://doi.org/10.1586/14737159.2015 .1112273

[11] LEKCHNOV EA, ZAPOROZHCHENKO IA, MOROZKIN ES, BRYZGUNOVA OE, VLASSOV VV et al. Protocol for miRNA isolation from biofluids. Anal biochem 2016; 499: 78-84. https://doi.org/10.1016/j.ab.2016.01.025

[12] ZHAO G, HAN C, ZHANG Z, WANG L, XU J. Increased expression of microRNA-31-5p inhibits cell proliferation, migration, and invasion via regulating $\mathrm{Sp} 1$ transcription factor in HepG2 hepatocellular carcinoma cell line. Biochem Biophys Res Commun 2017; 490: 371-377. https://doi. org/10.1016/j.bbrc.2017.06.050

[13] IGARASHI H, KURIHARA H, MITSUHASHI K, ITO M, OKUDA $\mathrm{H}$ et al. Association of MicroRNA-31-5p with Clinical Efficacy of Anti-EGFR Therapy in Patients with Metastatic Colorectal Cancer. Ann Surg Oncol 2015; 22: 2640 2648. https://doi.org/10.1245/s10434-014-4264-7

[14] CHEUNG CC, CHUNG GT, LUN SW, TO KF, CHOY KW et al. miR-31 is consistently inactivated in EBV-associated nasopharyngeal carcinoma and contributes to its tumorigenesis. Mol Cancer 2014; 13: 184. https://doi.org/10.1186/14764598-13-184

[15] WU J, TAN X, LIN J, YUAN L, CHEN J et al. MinicircleoriP-miR-31 as a Novel EBNA1-Specific miRNA Therapy Approach for Nasopharyngeal Carcinoma. Hum Gene Ther 2017; 28: 415-427. https://doi.org/10.1089/hum.2016.136

[16] LAI YH, LIU H, CHIANG WF, CHEN TW, CHU LJ et al. MiR-31-5p-ACOX1 Axis Enhances Tumorigenic Fitness in Oral Squamous Cell Carcinoma Via the Promigratory Prostaglandin E2. Theranostics 2018; 8: 486-504. https:/doi. org/10.7150/thno.22059

[17] ESQUELA-KERSCHER A, SLACK FJ. Oncomirs - microRNAs with a role in cancer. Nat Rev Cancer 2006; 6: 259-269. https://doi.org/10.1038/nrc1840

[18] BRAICU C, RADULY L, MORAR-BOLBA G, COJOCNEANU R, JURJ A et al. Aberrant miRNAs expressed in HER-2 negative breast cancers patient. J Exp Clin Cancer Res 2018; 37: 257. https://doi.org/10.1186/s13046-018-0920-2

[19] BERINDAN-NEAGOE I, MONROIG PDEL C, PASCULLI B, CALIN GA. MicroRNAome genome: a treasure for cancer diagnosis and therapy. CA Cancer J Clin 2014; 64: 311-336. https://doi.org/10.3322/caac.21244

[20]. QU K, LIN T, PANG Q, LIU T, WANG Z et al. Extracellular miRNA-21 as a novel biomarker in glioma: Evidence from meta-analysis, clinical validation and experimental investigations. Oncotarget 2016; 7: 33994-34010. https://doi. org/10.18632/oncotarget.9188

[21] ZHANG J, SONG Y, ZHANG C, ZHI X, FU H et al. Circulating MiR-16-5p and MiR-19b-3p as Two Novel Potential Biomarkers to Indicate Progression of Gastric Cancer. Theranostics 2015; 5: 733-745. https://doi.org/10.7150/ thno. 10305 